\title{
Hacia una definición de país forestal: ¿Dónde se sitúa Chile?
}

Towards a definition of a forest country: Where is Chile located?

\author{
PABLO J. DONOSO ${ }^{1}$, LUIS A. OTERO ${ }^{2}$ \\ ${ }^{1}$ Universidad Austral de Chile, Instituto de Silvicultura, Casilla 567, Valdivia, Chile. E-mail pdonoso@uach.cl \\ ${ }^{2}$ Centro Estudios Transdisciplinarios y Ambientales (CEAM), Universidad Austral de Chile, Casilla 567, \\ Valdivia, Chile. E-mail: lotero@uach.cl
}

\begin{abstract}
SUMMARY
Since the arrival of Spaniards, Chile has had great changes in its forestry landscape, mainly during the $19^{\text {th }}$ century. Land clearance for cattle and agriculture was originally the main cause of forest losses. The period of indiscriminate destruction of forests corresponds to the first stage of "Exploitation" of the forest resources according to the classification by Kimmins to describe the degree of forest development. In Chile this stage ended with the last great destruction of forests in Aysén, during the middle part of the $20^{\text {th }}$ century. According to Kimmins the first stage is followed by one of "Regulation", which is characterized by the creation of political and legal mechanisms to control forest harvest. Chile enters this stage, through state initiatives, with the creation of the institutional and industrial bases, by the creating the Corporación Nacional Forestal (Chilean Forest Service), the INFOR (Forest Research Institute), and the pulp mills Arauco and Constitución. The third stage corresponds to that of "Sustainable Forest Management", in which there is a sustained provision of goods and services from the forest. In this article our objective is to conduct a critical analysis to determine whether Chile has reached this stage of development, and for that purpose we established three requisites that Chile should fulfill to consider itself a developed nation from a forestry perspective: a) a great proportion of the population must improve their quality of life through the goods and services provided by the forests; b) strong public and private institutions should safeguard the good management of plantations and native forests, and; c) plantations and native forests are carefully managed so as to conserve or improve their biodiversity, productivity and the ecosystem services that they can provide. Through our analysis we conclude that these conditions are not met in Chile, and the pending challenge is to move the Chilean forest model towards greater levels of sustainability, particularly in social and environmental matters, in order become a nation with a truly developed forest sector.
\end{abstract}

Key words: forestry development, plantations, native forests, sustainable forest management.

\section{RESUMEN}

Desde la llegada de los españoles Chile ha sufrido grandes cambios en el paisaje forestal, particularmente a partir del siglo XIX. Las grandes pérdidas de bosques fueron causadas originalmente por la apertura de tierras para la agricultura y la ganadería. El período de destrucción indiscriminada de los bosques corresponde a la primera Etapa de "Explotación" de los recursos de acuerdo a la clasificación establecida por Kimmins, para describir el nivel de desarrollo forestal de los países. Se puede considerar que en Chile esta fase terminó luego de la última gran destrucción de bosques en Aysén, a mediados del siglo XX. Según Kimmins a la primera Etapa le sigue una de "Regulación", caracterizada por la creación de mecanismos políticos y legales para controlar la cosecha de bosques. Chile entra a esta Etapa con la creación por parte del Estado de las bases de la institucionalidad y la industria forestal chilena, en donde son ejemplos emblemáticos la creación de la Corporación Nacional Forestal (CONAF), el Instituto Forestal (INFOR), y las empresas de pulpa Arauco y Constitución. La tercera Etapa corresponde a la de "Desarrollo Forestal Sustentable", aquella en que existe una oferta sostenible de bienes y 
servicios de los bosques. En este artículo nuestro objetivo es hacer un análisis crítico para determinar si Chile ha alcanzado esta Etapa, para lo cual establecimos tres requisitos que deberían cumplirse para que Chile pueda considerarse un "País Forestal": a) una gran parte de la población debe ver mejorada su calidad de vida a través de los bienes y servicios provenientes de los bosques; b) existe una institucionalidad fuerte, tanto pública como privada, que resguarda el cumplimiento de normas modernas que regulan el buen manejo de plantaciones y bosques nativos, y c) las plantaciones y los bosques nativos son cuidadosamente manejados, de modo de conservar o mejorar la biodiversidad, la productividad y los servicios ecosistémicos que éstos proveen. A través de nuestro análisis concluimos que en su conjunto estas condiciones no se cumplen en Chile, y que el desafío pendiente es conducir el modelo forestal chileno hacia mayores niveles de sustentabilidad, particularmente en lo ambiental y social, para poder decir que Chile es realmente un "País Forestal".

Palabras clave: desarrollo forestal, plantaciones, bosque nativo, manejo forestal sustentable.

\section{INTRODUCCION}

Las naciones nuevas establecidas en zonas de bosques se han caracterizado por la apertura de tierras para la agricultura y la ganadería, sobre la base de la corta de estos bosques. Una vez habilitadas estas tierras, la evolución con respecto al tipo de uso de los bosques y la relación de la sociedad con los bosques ha sido variable. En Chile, desde la llegada de los españoles el paisaje forestal ha sufrido grandes cambios, debido a que la mayoría de la población se ha establecido en regiones de climas y bosques mediterráneos o templados, muy aptos para la agricultura y la ganadería. Kimmins (1) señala que la evolución forestal de los países generalmente se puede explicar en cuatro etapas, desde la más primitiva de explotación hasta una superior, caracterizada por un uso de los bosques altamente responsable, tanto desde el punto de vista ecosistémico como social (cuadro 1). A nuestro juicio, la clasificación de Kimmins (1) facilita un análisis de la evolución forestal de Chile, y particularmente de la situación actual del sector forestal.

Entre la primera mitad del siglo XIX y mediados del siglo XX, Chile se caracterizó por el aumento poblacional, la subdivisión de tierras y la destrucción de grandes extensiones de bosques para el desarrollo de la agricultura y la ganadería. Esta es la Etapa de Explotación (cuadro 1), y puede considerarse que culmina con la destrucción de cerca de 3 millones de hectáreas producto de la colonización de Aysén. Es tal vez la promulgación de la Ley de Bosques, en 1931 (que no se aplicó realmente hasta la segunda mitad del siglo), el primer anuncio de una transición hacia la Etapa 2, la de "Regulación" de las actividades forestales, y que se fortalece con la promulgación del Decreto
Ley 701 de fomento forestal (1974), con el establecimiento de la Tipología Forestal (2), y el Decreto Supremo 259, que establece regulaciones para la corta en bosque nativo. Adicionalmente se comienza a formar la institucionalidad forestal del país, con la creación de las primeras escuelas de ingeniería forestal en los años 50, y con posterioridad de la Corporación Nacional Forestal (CONAF), el Instituto Forestal (INFOR) y del Sistema Nacional de Areas Silvestres Protegidas del Estado (SNASPE), aunque este último aún no tiene un cuerpo legal que lo respalde. A partir de la segunda mitad del siglo XX, también hay un aumento notable de la participación de pino insigne (Pinus radiata) en la producción anual de madera aserrada (de un 3\% en los años 30 a un $85 \%$ durante los últimos años), y el Estado invierte en forestación y en la creación de las plantas de celulosa en los años 60 y 70 en Arauco y Constitución. En la Etapa de Regulación el Estado se constituye en el pilar del crecimiento del subsector de plantaciones con pino insigne, primero en forma directa y luego, con la promulgación del DL 701, en forma subsidiaria.

Terminado el siglo XX, Chile posee un sector forestal asimétrico: cuenta con 13,44 millones de hectáreas de bosques nativos y 2,12 millones de hectáreas de plantaciones (3), donde las primeras están parcialmente protegidas en el SNASPE, pero mayoritariamente sometidas a cosechas no controladas de maderas, cuyo destino es principalmente la leña, mientras que las plantaciones (pino y eucalipto) son la base de un subsector económico forestal que ha crecido rápidamente, que representa un 3,5\% del Producto Interno Bruto Nacional y un $13 \%$ de las exportaciones. Algunos cambios importantes ocurridos durante los últimos años incluyen el que algunas empresas hayan 


\section{CUADRO 1}

Descripción de las cuatro etapas de la evolución forestal (adaptado de Kimmins, 1997).

Description of the four stages of forest evolution (adapted from Kimmins, 1997).

Etapa 1: Explotación. Consiste en la explotación no regulada de bosque, que conduce con el tiempo a escasez de madera, leña y otros bienes. Aquí no hay silvicultura ni manejo forestal. La explotación de las maderas más valiosas y la quema de grandes superficies de bosques son la tónica común en esta etapa. La madera tiene escaso valor y el bosque es visto en general como un estorbo para el desarrollo de los países, particularmente para la ampliación de la frontera agrícola. La industria forestal es incipiente y rudimentaria, hay abundancia de leña y se extienden los planes de colonización agropecuaria.

Etapa 2: Regulación. Se institucionalizan mecanismos políticos y legales, se establecen regulaciones para controlar la tasa y los patrones de explotación forestal, de modo de asegurar el abastecimiento futuro de productos forestales. Este es el inicio del manejo forestal, que incluye una aproximación administrativa, centralizada, basada en la legislación y la regulación. Aunque existe el conocimiento respecto a cómo responden los bosques al manejo, y de cómo los ecosistemas forestales funcionan, en los hechos las actividades forestales, particularmente en bosques nativos, no son ecológica y ni silviculturalmente sostenibles. En esta etapa normalmente se desarrolla una silvicultura simple, basada en plantaciones y en la viverización de unas pocas especies. Algunos individuos y autoridades adquieren mayor conciencia de los problemas ambientales generados por la devastación de los bosques, la erosión, la pérdida de biodiversidad y de los paisajes y como respuesta a estas necesidades se crean grandes cantidades de áreas protegidas bajo administración del Estado, las que muchas veces no se desarrollan y cumplen sólo algunas de sus funciones.

Etapa 3: Manejo Forestal Sustentable. Esta incluye un enfoque ecológico en la aplicación de la silvicultura y el manejo forestal, tanto de los bosques nativos como de las plantaciones. Si bien el principal objetivo sigue siendo el producir madera, esto se hace de modo ambientalmente correcto, y asegurando un abastecimiento sostenido de madera y otros bienes y servicios de los bosques. El reciente impulso a los procesos de certificación forestal a nivel mundial está orientado hacia este tipo de manejo (e.g., ISO 14001, el sistema Pan Europeo (PEFC) y el Forest Stewardship Council (FSC)).

Etapa 4: Forestería Social. En esta etapa la conexión entre los intereses societales y de comunidades locales con las actividades forestales es fuerte, y las decisiones respecto al uso de los bosques son conjuntas y consensuadas con los propietarios de éstos. El rol del Estado es menor y los bosques cumplen sus funciones sociales y ambientales sin necesidad de presiones de mercado o de tipo legal. Además, los bosques son parte de la cultura y de los derechos colectivos.

optado por el manejo sustentable impulsadas especialmente por la certificación forestal que promueve el Forest Stewardship Council (FSC) (4), el surgimiento de iniciativas de conservación o protección privada, y el refinamiento y la diversificación de productos, entre otras.

Desde hace un par de décadas los empresarios de la madera y sucesivos gobiernos a través de sus instituciones forestales han acuñado la expresión de "Chile, País Forestal". Si bien las características de Chile claramente permiten identificarlo como un país de vocación forestal, en este artículo queremos analizar críticamente si, a la luz de su situación forestal actual en aspectos económicos, sociales e institucionales, Chile tiene los méritos suficientes para autocalificarse como País Forestal, y para ello nuestro énfasis está en observar el sector desde una perspectiva de desarrollo (la realización de un mayor y más completo potencial), y no sólo de crecimiento (incremento en tamaño por medio de crecimiento material) (sensu 5), que es la tendencia dominante de la economía actual. Es decir, sugerimos que además de poder mostrar cifras exitosas con respecto al crecimiento del subsector plantaciones y de superficies de bosques, se debe analizar si dicho crecimiento se condice con el mejoramiento de las condiciones sociales y ambientales 
del entorno donde ocurren las actividades forestales.

Para ello se plantea que para que legítimamente un país pueda denominarse País Forestal debe haberse alcanzado la Etapa 3 de Manejo Forestal Sustentable de Kimmins (o similarmente deben satisfacerse los indicadores de manejo forestal sustentable del Criterio 7 de la Declaración de Santiago), lo que a nuestro juicio se puede simplificar en los tres siguientes grandes requisitos:

a) una gran parte de la población que vive en las zonas forestales del país ve mejorada su calidad de vida a través de los bienes y servicios provenientes de los bosques;

b) existe una institucionalidad fuerte tanto pública como privada (ONGs, asociaciones de propietarios, grupos científicos, etc.) que resguarda el cumplimiento de normas modernas que regulan el buen manejo de plantaciones y bosques nativos, así como la conservación de estos últimos;

c) las plantaciones y los bosques nativos son cuidadosamente manejados de modo de conservar o mejorar la biodiversidad, la productividad y los servicios ecosistémicos que éstos proveen.

El esquema sugerido por Kimmins es apropiado debido a que considera no sólo aspectos relativos a la sustentabilidad de prácticas ambientales a nivel de bosque e industria, sino que también aspectos institucionales y sociales, esto último reflejado, por ejemplo, en la inclusión de la Etapa de Forestería Social. Esta aproximación al análisis de la sustentabilidad forestal es consistente con el Criterio 7 de la Declaración de Santiago, que siguió al Proceso de Montreal, y que fue firmada por Chile junto a 11 países de zonas templadas (excluido Europa que había firmado el llamado Proceso de Helsinki), la cual considera una serie de indicadores ambientales, sociales, institucionales y económicos para evaluar el manejo forestal sustentable de los países signatarios.

REQUISITO 1: CALIDAD DE VIDA DE LA POBLACION EN LAS ZONAS FORESTALES

Una de las mayores contradicciones del sector forestal chileno es que mientras ha habido un crecimiento notable de las exportaciones forestales a partir de plantaciones de pino y eucalipto princi- palmente distribuidas en las Regiones VIII y IX $(6,7)$, la población de esas Regiones no ha visto mejorada su calidad de vida. Durante el rápido proceso de expansión de las plantaciones (principalmente durante los 70 y 80 ) ocurrieron problemas de migraciones, crecimiento explosivo de pueblos bien establecidos y de desempleo (8). En la actualidad la actividad forestal en Chile se concentra desde las Regiones VII hasta X, y son justamente estas cuatro Regiones las que tienen los Indices de Desarrollo Humano (IDH) más bajos (9). Según datos del gobierno (10), entre 1990 y 1998 las Regiones VIII y IX han sido aquellas con mayor cantidad de pobres $(40,5 \%$ en promedio en 1990 y $27,3 \%$ en promedio en 1998) e indigentes $(15,2 \%$ promedio en 1990 y $8,5 \%$ promedio en 1998). De acuerdo a la misma fuente, la relación entre pobreza y distribución del ingreso es evidente de acuerdo al Coeficiente de Gini: en 1998 las Regiones VIII y IX presentaban las peores condiciones de distribución del ingreso en Chile. Por áreas particulares, la IX Región es aquella con un mayor porcentaje de gente que recibe salud entre regular y muy mala $(41,7 \%)$, y en educación, las Regiones VII, X y IX son aquellas con mayor porcentaje de población entre 4 y 17 años que no asiste a un establecimiento educacional. Más aún, la VIII Región, que concentra las mayores superficies de plantaciones de especies exóticas, es también la más pobre y aquella con el porcentaje de población rural indigente más alto del país. Luego de la VIII Región, la IX Región es aquella de mayor actividad forestal en Chile y la de mayor concentración de población Mapuche. Los Mapuche en esta Región perciben la mitad de los ingresos y sus IDH en todos los aspectos son inferiores a los de la gente no Mapuche (11). Adicionalmente, en la actualidad existen severos conflictos entre empresas forestales o agrícolas y comunidades Mapuche por reclamos relativos a propiedad de tierras (12).

Aunque sería injusto atribuir los problemas señalados exclusivamente al sector forestal, los datos señalados sugieren al menos que éste no ha contribuido a mejorar la situación socioeconómica de los habitantes de estas regiones, ni siquiera a nivel rural. Para ilustrar, entre los años 1987 y 1996 las Regiones VII, VIII y IX, además de la III, fueron las que registraron peores índices de superación de la pobreza (13), mientras que las exportaciones forestales durante el período fueron 
en ascenso (14). Esto se explica, entre otros factores, por los bajos sueldos de los trabajadores, factor que justamente ha ayudado a atraer capitales al sector forestal chileno (15).

En el aspecto cultural, al compararse Chile con una potencia mundial forestal como Suecia (probablemente un país en la Etapa 4 de Forestería Social de Kimmins), cuyas exportaciones forestales son cinco veces superiores, hay varios datos que reflejan diferencias con respecto al uso de los bosques por parte de la ciudadanía. Con respecto a Chile, en Suecia: a) es mucho mayor el consumo de papel per cápita (246 vs. alrededor de 50 kg per cápita al año) y el uso de madera para construcciones $\left(0,5 \mathrm{~m}^{3}\right.$ vs. menos de $0,1 \mathrm{~m}^{3}$ per cápita al año) $(16,17)$; b) existe el derecho al libre acceso a los bosques ("prerrogativa al acceso común" o "Allemansrätt"; 18, 19), a través de lo cual la población recolecta frutos silvestres y hongos (entre 30 y 15 millones de litros al año, respectivamente); c) existe un plan para reducir y cerrar sus centrales nucleares, las que serán reemplazadas por dendroenergía, entre otras alternativas (en Suecia poco más del $20 \%$ de la energía industrial se genera con biomasa, la mayoría proveniente de desechos del bosque). Como contraste, en Chile, con una superficie de bosque per cápita relativamente abundante y comparable con la de países forestalmente desarrollados, la población no tiene acceso libre a los bosques, no está integrada a éstos, y no hay ninguna política dendroenergética, aunque Chile aceptó la obligación internacional voluntaria de reemplazar la energía fósil por energía renovable en la Convención de Cambio Climático (20). De hecho, en Chile el consumo industrial de leña es muy bajo pero a nivel doméstico cubre cerca del $20 \%$ de las necesidades energéticas de la población, a través de mercados informales en los cuales no se tributa (20).

En resumen, a pesar de haber 117 mil personas ocupadas en el sector forestal chileno $(2,1 \%$ del total) los bosques no son parte de la vida cotidiana de los chilenos, y más aún una mayoría de la población está cada vez más desconectada de los bosques, en la medida que aumenta la concentración de la propiedad forestal, la migración rural-urbana y el uso de materiales no madereros en la construcción.
REQUISITO 2: INSTITUCIONALIDAD, EDUCACION E INVESTIGACION FORESTAL

Institucionalidad. El Estado de Chile tiene un par de instituciones forestales de larga trayectoria y consolidadas: la CONAF y el INFOR. La CONAF tiene como misión la conservación, protección, incremento, manejo y aprovechamiento de los recursos naturales renovables del país, y sus funciones principales son aplicar la legislación forestal, administrar y cuidar las Areas Protegidas del Estado (a través del SNASPE), y controlar los incendios y plagas forestales. El INFOR tiene como misión investigar y transferir conocimientos científicos y tecnológicos sobre los recursos forestales, y por lo tanto se verá más adelante, en la sección de investigación.

La CONAF es y ha sido reconocida nacional e internacionalmente como órgano de importancia en materia forestal. Según Fernández (21), incluso comercialmente la CONAF es una marca que se ha ganado un lugar en el mundo forestal, cualesquiera que hubiesen sido sus desaciertos en esta materia. Sin embargo, en la actualidad se ve sobrepasada para cumplir estas funciones. En cuanto a fiscalización, su debilidad se ve reflejada en que sólo entre 5 y $25 \%$ de los bosques nativos intervenidos son afectos a silvicultura o buen manejo $(22,23)$. En cuanto al SNASPE, si bien cubre un $19 \%$ y del territorio nacional y un $29 \%$ de los bosques nativos, éste no es suficientemente representativo de la diversidad de los ecosistemas chilenos, está muy lejos de cumplir la meta de conservar al menos un $10 \%$ de cada ecosistema significativo al año 2010 , y no representa a 17 de las 85 formaciones vegetales del país $(24,25)$. Con respecto a los incendios forestales, su número y superficie afectada han aumentado en un $20 \%$ durante la última década, y ha habido dos incendios de gran envergadura que han dañado ecosistemas de alto valor (once mil ha en el Parque Nacional Torres del Paine el 2005, y siete mil ha en el PN Tolhuaca en el 2002). El reciente informe de OCDE-CEPAL (24) señala, en el caso de CONAF, que la labor de hacer cumplir las normas de protección de la naturaleza en sí mismas parece ser el pariente pobre de las actividades centrales de desarrollo de la entidad.

La debilidad institucional forestal de Chile queda también de manifiesto al compararse esta 
con la de otros países templados de importancia forestal en el mundo. Evaluando los indicadores del Criterio 7 de la Declaración de Santiago, Sedjo et al. (15) consideran que el marco legal, institucional y económico requerido para impulsar un desarrollo forestal sustentable está bien desarrollado en todos los países evaluados (Canadá, Estados Unidos, Francia, Alemania, Finlandia, Nueva Zelandia y Suecia) con excepción de Chile, donde es considerado moderado. Similar situación ocurre con la evaluación de la habilidad del marco institucional para aplicar adecuadamente las políticas o leyes forestales. Adicionalmente, Chile es el único de estos países mencionados que no ha generado o actualizado leyes forestales durante los últimos 15 años, con la finalidad de que éstas promuevan un manejo forestal que resguarde la biodiversidad y otros servicios ecosistémicos. Un ejemplo emblemático es el del proyecto de Ley de Recuperación y Manejo de los Bosques Nativos, que se discute en el Congreso de Chile desde 1992, sin que éste logre ser aprobado hasta la fecha, principalmente debido a la insistencia de algunos sectores en mantener abierta la posibilidad de sustitución de las especies nativas por plantaciones de exóticas, cuestión que también es muy inconveniente para la imagen de un país exportador de productos forestales como el nuestro.
Educación. Chile posee dos escuelas de ingeniería forestal de más de 50 años, aquellas de la Universidad de Chile y de la Universidad Austral de Chile, las que a través de la creación temprana de ingenieros forestales fueron fundamentales para que se estableciera la institucionalidad y la industria forestal en el país. Sin embargo, en las últimas décadas se ha creado una serie de otras escuelas, lo que ha llevado a que en la actualidad Chile muestre una gran desproporción entre el número de escuelas de ingeniería forestal con respecto a la superficie de bosques y la población del país. Mientras que en países desarrollados existe del orden de una escuela forestal por cada 4-10 millones de habitantes, en Chile hacia finales del año 2004 existía aproximadamente una por cada 1,5 millones de habitantes (cuadro 2). Similarmente, en Chile hay una escuela forestal por cada 1,5 millones de ha, mientras que esta relación en los otros países es de una escuela por cada 7-13 millones de ha, con la excepción de Canadá donde la relación es de una escuela por cada 33 millones de hectáreas. Como consecuencia, en Chile, según el Ministerio de Educación, de las universidades chilenas que imparten ingeniería forestal el año 2001 egresaron 111 personas y se titularon 274 (26).

Actualmente la percepción generalizada es que hay una gran cantidad de ingenieros forestales que

\section{CUADRO 2}

Relaciones entre número de escuelas forestales, población (millones) y superficie de bosques (millones de ha) en algunos países con importante actividad forestal ${ }^{\mathrm{a}}$.

Relationships between number of forest schools, population (millions) and forest cover (millions of ha) in selected countries with an important forest activity.

\begin{tabular}{|c|c|c|c|c|c|c|}
\hline País & Población & $\begin{array}{l}\text { Superficie } \\
\text { forestal } \\
\text { (2) }\end{array}$ & $\begin{array}{c}\mathrm{N}^{\circ} \text { escuelas } \\
\text { forestales } \\
\text { (3) }\end{array}$ & $\begin{array}{l}\text { Superficie } \\
\text { forestal per } \\
\text { cápita } \\
\text { (1):(2) }\end{array}$ & $\begin{array}{l}\text { Superficie de } \\
\text { personas vs. } \\
\text { escuelas } \\
\text { forestales } \\
\text { (1):(3) }\end{array}$ & $\begin{array}{l}\text { Bosques vs. } \\
\text { escuelas } \\
\text { forestales } \\
\text { (2):(3) }\end{array}$ \\
\hline Chile & 15 & 15 & 10 & 1,0 & 1,5 & 1,5 \\
\hline EE.UU. & 267 & 212 & 24 & 0,79 & 11,1 & 9,09 \\
\hline Suecia & 9 & 24 & 2 & 2,66 & 4,55 & 12,5 \\
\hline Finlandia & 5 & 20 & 2 & 4,00 & 2,50 & 10,0 \\
\hline Canadá & 30 & 244 & 7 & 8,13 & 4,35 & 33,3 \\
\hline Nueva Zelandia & 4 & 8 & 1 & 2,0 & 4,00 & 7,69 \\
\hline
\end{tabular}

a: Datos a partir de diversas fuentes en Internet. 
no están trabajando en el rubro forestal o que simplemente están desempleados, escenario difícil de cambiar mientras siga habiendo, entre otras variables, un exceso de carreras de ingeniería forestal. Por otra parte, se da el absurdo de que el país carece de escuelas de formación para obreros forestales especializados, para preparar motosierristas, operadores de maquinaria de cosecha, capataces en silvicultura, etc. La única escuela existente, el Centro Nacional de Capacitación Forestal de Escuadrón, en Concepción, fue cerrada hace más de 10 años. Hoy día las empresas forestales deben capacitar a su propio personal a través de sistemas privados. La pirámide de formación está invertida, es más fácil encontrar un ingeniero forestal que un motosierrista capacitado y, a pesar de que hoy existen iniciativas de certificación laboral, no existen políticas al respecto por parte del Estado.

Investigación. Benedetti y Saavedra (27) señalan que la orientación actual del Estado es financiar investigación en la medida que ésta contribuya al desarrollo e innovación tecnológica para fomentar el crecimiento económico y social del país. La investigación se financia a partir de recursos concursables, colaboración de privados, investi- gación precompetitiva y competitiva, y debe asegurar la rentabilidad de negocios que se generen a partir de los resultados (27). A partir de esta filosofía la Corporación de Fomento (a través de los fondos FIA, FDI y FONTEC) hasta el año 2003 había entregado 17.2 mil millones de pesos al sector forestal, es decir, un promedio de 2,87 mil millones anuales. Estos fondos han sido destinados principalmente a las áreas de Silvicultura e Industria y Tecnología, pero una mínima parte a bosques nativos (27). Adicionalmente, el aporte de la Comisión Nacional de Investigación, Ciencia y Tecnología (CONICYT) para la investigación de los bosques nativos, a través de FONDEF y FONDECYT, alcanza a menos de un $2 \%$ del monto total asignado a investigación (cuadro 3), muy similar a la situación de principios de los años 90 (28).

El INFOR debe competir por el financiamiento para la investigación al igual que cualquier otra institución. Es decir, el INFOR no puede responder, a través de la investigación, a las necesidades de investigación forestal del país, en primer lugar porque debe desgastarse en competir por los recursos, y en segundo lugar porque no posee importantes predios experimentales forestales donde sus profesionales puedan conducir investigación

\section{CUADRO 3}

Asignación de dinero entre los años 2001 y 2004 para investigación a través de los programas FONDEF y FONDECYT Regular de CONICYT (en proyectos FONDECYT valores corresponden a aporte del año en el caso de Total y de tres años en el caso de Investigación Forestal y en Bosques Nativos; en el caso de proyectos FONDEF valores corresponden a aportes para el total del proyecto, generalmente tres años).

Money allocation from years 2001 through 2004 for forest research through the FONDEF and FONDECYT of CONICYT (in FONDECYT projects values correspond to annual budgets for the total, and three-year budgets in the cases of Forest Research and in Native Forests; in the case of FONDEF projects values correspond to budgets for the entire projects, generally three years).

\begin{tabular}{|lccccc|}
\hline Fondo & $\begin{array}{c}\text { Total } \\
\text { Miles \$) }\end{array}$ & $\begin{array}{c}\text { Investigación } \\
\text { forestal } \\
\text { (Miles \$) }\end{array}$ & $\begin{array}{c}\text { Investigación en } \\
\text { bosques nativos } \\
\text { (miles \$) }\end{array}$ & $\begin{array}{c}\text { Porcentaje } \\
\text { forestal vs. } \\
\text { total }\end{array}$ & $\begin{array}{c}\text { Porcentaje } \\
\text { bosque nativo } \\
\text { vs. total }\end{array}$ \\
\hline FONDECYT 2001 & 6.192 .223 & 16.862 & 10.484 & 0,82 & 0,51 \\
FONDECYT 2002 & 6.509 .927 & 43.169 & 43.169 & 1,99 & 1,99 \\
FONDECYT 2003 & 6.928 .160 & 50.573 & 43.119 & 2,20 & 1,87 \\
FONDEF 2001 & 10.190 .103 & 628.122 & 150.000 & 6,16 & 1,47 \\
FONDEF 2002 & 11.306 .000 & 1.443 .000 & 150.000 & 12,76 & 1,33 \\
FONDEF 2003 & 12.524 .000 & 1.090 .000 & 260.000 & 8,70 & 2,08 \\
FONDEF 2004 & 11.800 .000 & 388.000 & 0 & 3,30 & 0 \\
\hline
\end{tabular}


de largo plazo, como ocurre en países forestalmente desarrollados. A modo de ejemplo y contraste, en Estados Unidos de Norteamérica hay 61 estaciones forestales experimentales (29), en las cuales se desarrolla investigación de largo plazo que es publicada en revistas científicas de alto prestigio, así como también a través de documentos técnicos y de investigación en la página web del Servicio Forestal (Forest Service).

En resumen, a pesar de que en Chile hay una institucionalidad vigente desde hace décadas, que podría ser capaz de hacer respetar la legislación y regulaciones forestales y conducir el desarrollo forestal del país, y la educación e investigación forestal ya tienen medio siglo, la institucionalidad es débil, la educación forestal está desregulada y como consecuencia hay una mayor oferta que demanda de forestales, y existe una pobre asignación presupuestaria para investigar los recursos forestales del país.

REQUISITO 3: MANEJO DE PLANTACIONES Y BOSQUES NATIVOS PARA LA CONSERVACION DE LA BIODIVERSIDAD, DE LA PRODUCTIVIDAD Y DE LOS SERVICIOS ECOSISTEMICOS

El diseño de estrategias a través de las cuales los bosques sean diversos y provean la multiplicidad de bienes y servicios que la comunidad nacional e internacional demande de éstos es otro factor necesario para un desarrollo forestal sustentable (25). Para responder a esas demandas ha habido tradicionalmente dos aproximaciones (30): la de uso múltiple, en el cual cada bosque es usado simultáneamente para varios propósitos, y la de zonificación, en la cual distintos tipos de bosques son dedicados a distintas actividades (e.g., preservación $v s$. producción de madera). Una aproximación alternativa es la propuesta por Seymour y Hunter (31), en cuyo esquema incluyen tres tipos de bosques: 1) los bosques destinados a silvicultura de producción -principalmente plantaciones-; 2), bosques destinados a ser manejados bajo principios ecosistémicos (32), y 3), las reservas ecológicas. Aunque cada tipo de bosque tiene una función predominante distinta, éstos se manejan en un marco de conservación de la biodiversidad a nivel de región o de paisaje, de modo de conservar la biodiversidad local y asegurar la provisión de la diversidad de bienes y servicios demandados por la sociedad y comunidades locales de un modo sostenible en el tiempo. De acuerdo a este modelo, con excepción de las reservas ecológicas destinadas principalmente a preservación, tanto las plantaciones como los bosques nativos deberían proveer tanto bienes como servicios a la comunidad. Esta aproximación es más apropiada que las anteriores en el contexto actual en el cual la sociedad demanda crecientemente bienes y servicios de los bosques, y a la vez un uso ambiental y socialmente responsable de todos los ecosistemas forestales. Considerando esa última aproximación, a continuación se analiza de qué manera las plantaciones y los bosques nativos están proveyendo bienes y servicios en relación a su potencial.

Las plantaciones forestales y el manejo de bosques coetáneos. Las plantaciones forestales se están convirtiendo en forma creciente en la principal fuente de provisión de madera y papel en el mundo $(33 ; 34)$ y en Chile $(6,7)$. Mientras aumentan las plantaciones en el mundo, también aumentan las críticas negativas y positivas hacia este tipo de cultivos forestales.

Entre los aspectos negativos que se le atribuyen a las plantaciones está aquel de la pérdida de biodiversidad con respecto a aquella de bosques nativos, lo cual ha sido extensamente reportado en Chile (e.g., 35; 36; 37) y en otros bosques templados (e.g., 38; 39). Lo anterior obedece a que las plantaciones son monoespecíficas, de rotaciones cortas que permiten que éstas sólo alcancen un estado sucesional temprano (sensu 40), de especies exóticas, además de densas y regulares cuando el objetivo es producción de fibra, por lo que en ellas los niveles de transmisión de luz hacia el suelo y la diversidad estructural son menores que en bosques nativos (sensu 41), todo lo cual en definitiva causa la disminución de la diversidad de especies autóctonas (42).

Otro aspecto negativo atribuido a las plantaciones es que éstas disminuyen la productividad de los suelos por la erosión asociadas a sus sistemas de cosecha y por la alta demanda de nutrientes del suelo, los que no se recuperan durante las rotaciones cortas con que son manejadas. Con las talas rasas con las que se cosechan las plantaciones en Chile, las que a veces llegan a más de 100 ha, se generan cambios drásticos en el microclima, aceleramiento de los procesos erosivos y pérdida de nutrientes (sensu 43, 44), lo cual en el largo plazo disminuye la productividad del sitio. 
Para evitar los efectos ambientales negativos descritos arriba, durante las etapas de cosecha y regeneración se deberían tomar las siguientes medidas, muchas de las cuales son parte de las Prácticas de Buen Manejo (Best Management Practices) que se exigen en muchas regiones forestales del mundo: 1) planificar cuidadosamente los caminos y las vías de madereo, evitando que éstos cubran más del $15 \%$ del área, ya que en estos sectores la erosión y compactación del suelo son más críticas; 2) continuar con la política implementada por algunas empresas de eliminar las quemas controladas de modo de conservar los nutrientes acumulados en la biomasa y disminuir la erosión, así como de evitar la muerte directa de una gran cantidad de vida silvestre, la eliminación de sitios de nidificación, y los efectos negativos sobre el paisaje; 3 ) hacer uso de los conocimientos de manejo vegetacional integrado, es decir, el manejo de la vegetación competidora en función del logro de objetivos silviculturales usando una variedad de métodos que sean ambientalmente responsables y social y económicamente aceptables (45); 4) establecer un irrestricto respeto a la vegetación nativa existente en las quebradas; 5) realizar retenciones estructurales que permitan mantener los nichos más importantes para la biodiversidad incluyendo los "árboles percha", material leñoso muerto y bosquetes de vegetación nativa (sensu 32); 6) realizar plantaciones de baja densidad o realizar raleos tempranos que permitan la incorporación de otras especies al bosque o de la propia regeneración natural de especies nativas, que pueden acompañar a la especie plantada; 7) disminuir el tamaño de las talas rasas o utilizar otras técnicas igualmente eficientes como talas en fajas o en claros, las que permiten mantener un dosel de protección lateral, y 8) realizar nuevos diseños para las plantaciones, siguiendo las curvas de nivel y evitando los bordes de las plantaciones rectas, que empobrecen el paisaje de las zonas turísticas del sur del país.

Entre los beneficios ecológicos que se le reconocen a las plantaciones, uno de carácter indirecto es que éstas pueden reducir la presión sobre la cosecha de bosques nativos (46) y facilitar que más bosques nativos puedan ser destinados a preservación o manejados con horizontes de más largo plazo $(1,47)$. Si bien en Chile ha disminuido notablemente el uso industrial de maderas nativas debido a la alta oferta de pino, la cual ha bajado la presión por estas maderas, se estima que por políticas erradas que autorizaban la sustitución, tras el establecimiento de plantaciones ha ocurrido una pérdida de más de 200.000 ha $(25 ; 47)$, y la grave fragmentación de ecosistemas forestales nativos, siendo la situación más preocupante aquella de los bosques maulinos (48). Aunque las tasas más fuertes de sustitución de bosques ocurrieron en las décadas de los 70 y 80, en los 90s aún se señalaba que entre un 3 y $6 \%$ de las plantaciones eran establecidas tras sustituciones de bosques nativos (15).

El segundo beneficio, de carácter directo, tiene que ver con que las plantaciones pueden contribuir a la disminución del efecto invernadero por su capacidad de captura y almacenamiento de carbono atmosférico (e.g., protocolo de Kyoto -1; 49; 50). Este punto es especialmente relevante considerando que el incremento en la temperatura global de $1.5-4.5^{\circ} \mathrm{C}$ esperado para mediados del presente siglo generará migraciones de ecosistemas, pérdidas de biodiversidad, impactos en la producción de alimentos y subidas en los niveles de los océanos $(51 ; 52)$. Sin embargo, a nuestro juicio este rol de las plantaciones es relevante sólo si éstas son establecidas y manejadas bajo las siguientes circunstancias:

a) Se permite que crezcan hacia un estado biológicamente más maduro, período durante el cual hay una mayor captura de carbono debido a la acumulación de biomasa viva y muerta en el ecosistema $(1 ; 37 ; 53)$;

b) Una buena parte de la madera producida se destina a productos con una larga vida útil (30 a 100 años; 52), o a energía (e.g., leña), que sustituye el uso de combustibles fósiles;

c) Las plantaciones forestales incrementan la superficie cubierta de bosques en una región.

Estos requisitos son sólo parcialmente cumplidos por las plantaciones forestales en Chile, ya que éstas se cosechan cuando jóvenes y cuando tienen aproximadamente sólo una tercera parte del máximo potencial de acumulación de carbono en el sistema, la mayor parte del carbono secuestrado por la plantación es rápidamente devuelto a la atmósfera (sensu 1) debido a que gran parte de la madera es destinada a celulosa y papel, cuya vida útil promedio es de sólo 1 y 5 años (54), y finalmente debido a que al menos un $10 \%$ de las plantaciones han reemplazado bosques nativos. 
Es difícil revertir el hecho de que la mayoría de las plantaciones se destinen a celulosa y papel, pero sí se podrían extender las rotaciones, limitar o modificar las talas rasas extensas en zonas de alta pluviometría, y definitivamente impulsar las plantaciones en suelos degradados, en función de incrementar su aporte ambiental. En este sentido deben valorarse las plantaciones establecidas en los suelos erosionados del secano interior de las Regiones VII y VIII, ya que éstas han tenido un efecto ambiental positivo al permitir la recuperación de los suelos y de su productividad. Similarmente, la Ley $\mathrm{N}^{\circ} 19.561$ de 1998 (continuación del DL 701) ha sido un paso positivo, por cuanto uno de sus énfasis es subsidiar plantaciones en suelos degradados.

El mejoramiento de prácticas de establecimiento y manejo de plantaciones, sumado a la diversificación de especies, incluyendo especies nativas, permitiría que las plantaciones puedan contribuir significativamente al paisaje, a la biodiversidad, a la reducción del efecto invernadero y a reducir la presión sobre los bosques nativos. Un paso importante para el manejo sustentable ha sido la incorporación de cerca de 500 mil hectáreas de bosques, en su gran mayoría plantaciones, al proceso de certificación FSC, que posee reconocimiento internacional y exigentes estándares sociales y ambientales. Desafortunadamente el Es- tado no ha asumido la certificación FSC como política oficial y ha promovido sellos locales, con exigencias insuficientes desde el punto de vista social y ambiental y con escaso reconocimiento internacional, como es el caso del sello CERTFOR de Fundación Chile.

Los bosques nativos y su manejo ecosistémico. Los bosques nativos en general tienen un alto potencial para sustentar varias de las funciones de los ecosistemas forestales descritas por de Groot et al. (55): regulación o mantención de los procesos ecológicos esenciales; provisión de hábitat para el crecimiento y la reproducción de plantas y animales; producción de bienes, y funciones de información tales como recreación y desarrollo cognitivo.

Una de las funciones ecológicas más relevantes que se les ha asignado mundialmente a los bosques nativos es la conservación de la biodiversidad. Los bosques nativos chilenos han cobrado gran relevancia internacional desde el punto de vista de la conservación de la biodiversidad, ya que: a) representan una porción importante de los escasos bosques templados del hemisferio sur y de los bosques costeros templados lluviosos del mundo (56); b) poseen un gran endemismo de flora y fauna (56); c) el SNASPE es deficiente en cuanto a la conservación de los más importantes ecosistemas terrestres presentes en el país

\section{CUADRO 4}

Crecimiento volumétrico de distintos tipos de bosques en Chile y en el mundo. Volume growth of different forests in Chile and the world.

\begin{tabular}{|lccccc|}
\hline \multirow{2}{*}{ Tipo Bosque } & \multicolumn{2}{c}{ Crecimiento medio } & \multicolumn{2}{c|}{ Crecimiento corriente } & \\
& $\mathrm{m}^{3} / \mathrm{ha} / \mathrm{año}$ & Edad & $\mathrm{m}^{3} / \mathrm{ha} / \mathrm{año}$ & Edad & Fuente \\
\hline Plantación Roble & $5,1-12,5$ & $12-14$ & - & - & 67 \\
Plantación Raulí & $4,8-13,1$ & $10-34$ & - & - & 67 \\
Plantación Coihue & $9,0-20,5$ & $9-18$ & - & - & 67 \\
Plantación Raulí & $15,0-17,1$ & 21 & $28-35$ & 21 & 65 \\
Renovales Raulí & $4,2-16,0$ & $30-50$ & - & - & $66 ; 68$ \\
Renoval Roble & 9,1 & 30 & 17,9 & $25-32$ & $64 ; 69$ \\
Renovales Coihue & $6-9$ & $20-27$ & $18-22$ & $20-27$ & 70 \\
Pinus radiata, VIII Región, Chile & $34,9-24,6$ & 25 & $20,5-8,1$ & 25 & 71 \\
& & & según sitio & & \\
Eucalipto, Chile & $20-45$ & $8-16$ & - & - & 72,73 \\
Boreal, Finlandia & $1,7-4,6$ & $80-120$ & - & - & 74 \\
Pinus taeda EE.UU. & 12 & 25 & - & - & 75 \\
\hline
\end{tabular}


$(24 ; 25 ; 58)$, y d) continúa habiendo tasas importantes de deterioro o destrucción de éstos $(23 ; 25$; 48). Por tales motivos, Chile es considerado hoy como uno de los 25 puntos críticos (hotspots) en el mundo en cuanto a la conservación de la biodiversidad $(59 ; 60)$. Aunque el incremento de las áreas protegidas privadas (que en pocos años alcanzan cerca de las $700 \mathrm{mil} \mathrm{ha}$ ) ha sido un aporte para la conservación de la biodiversidad, de acuerdo a Simonetti (61), esta estrategia no parece ser viable, ya que la conservación de la biodiversidad debe plantearse como una estrategia orientada a la matriz forestal del país y no sólo hacia la creación de islas protegidas, las que normalmente son difíciles de mantener y si llegan a alcanzar tamaños considerables pueden convertirse en un obstáculo para el desarrollo social y económico de las regiones. En este sentido, el manejo forestal sustentable debe ser la estrategia fundamental y las áreas protegidas deben ser sólo consideradas como un "complemento importante" (61).

Otro servicio ecosistémico relevante es el relativo a la capacidad de los bosques nativos de suministrar agua de calidad en abundancia y en forma relativamente regular durante todo el año, incluidos los períodos de verano (25). Esta capacidad de los bosques nativos es contrastante con las plantaciones de especies de rápido crecimiento, las que al ser altamente demandantes de agua pueden generar dramáticas reducciones de caudales durante los meses secos (62), así como cambios en los patrones de entrega de agua (25). Algunos estudios muestran pérdidas de producción de aguas en cuencas plantadas con Pinus radiata de un $28 \%$ (36). Estos resultados indican la urgencia de un manejo integrado de cuencas en el que el manejo de bosques no comprometa la producción de agua, especialmente en zonas con veranos secos.

El mal uso de los bosques nativos ha derivado en la degradación de éstos, con las consecuentes pérdidas asociadas en cuanto a servicios ecosistémicos y productividad de millones de hectáreas. Esto es particularmente lamentable en Chile, en donde la productividad de los bosques nativos está entre las más altas del mundo en bosques naturales (cuadro 3), la madera aserrada o para chapas de las especies principales tienen un valor entre tres y cinco veces superior a la de Pinus radiata (6) y la respuesta de estos bosques al manejo es positiva (e.g., diversos capítulos en $63 ; 64 ; 65)$.

En resumen, existe el potencial y la necesidad de hacer silvicultura y buen manejo de los bosques nativos en Chile no sólo en función de conservar su biodiversidad y obtener mayores beneficios de sus potenciales servicios ecosistémicos, sino que además porque se trata de bosques altamente productivos y valiosos que manejados harían una importante contribución social y económica al país.

\section{CONCLUSIONES}

El sector forestal chileno ha tenido un significativo avance durante los últimos 50 años. Chile hoy cuenta con instituciones forestales, carreras de ingeniería forestal y un SNASPE de larga trayectoria, y en general su importancia forestal es reconocida internacionalmente. Sin embargo, la aún débil institucionalidad forestal, la deuda social del sector forestal y las aún limitadas iniciativas de manejo forestal sustentable, particularmente en sus bosques nativos, indican que el sector forestal de Chile aún no supera la etapa de Regulación, es decir, aquella en que el país posee una multiplicidad de regulaciones forestales y ambientales y ha creado una gran cantidad de áreas silvestres protegidas, pero en que estas medidas no son suficientes para asegurar la conservación de la biodiversidad de sus ecosistemas, revertir el proceso de pérdida y degradación de bosques y ecosistemas forestales, y fomentar un manejo eficiente y con perspectivas ecosistémicas de estos bosques. De hecho, al compararse con otros países de importancia forestal (Canadá, EE.UU., Nueva Zelandia, Alemania, Francia, Suecia y Finlandia), Chile aparece como un país que sufriría en promedio los impactos más fuertes si se le aplicaran políticas de forestería sustentable reconocidas internacionalmente (15).

El desafío forestal es que Chile avance hacia Etapa 3, la de Manejo Forestal Sustentable, resguardando que el manejo de los bosques nativos y de las plantaciones sea responsable ambiental y socialmente. A nuestro juicio, sólo entonces sería legítimo reconocer o calificar a Chile como País Forestal. El mejoramiento de la situación actual respecto al mal uso de los bosques, la superación de los problemas sociales que ha generado el cre- 
cimiento del sector forestal y el necesario fortalecimiento institucional forestal que requiere Chile, dependen importantemente de que exista la voluntad política necesaria para catalizar los cambios requeridos. El que ocurra esto es incierto, particularmente cuando en Chile se sigue promoviendo el crecimiento económico sin una perspectiva clara de desarrollo sustentable, y se sigue optando por un modelo forestal concentrador y por lo tanto excluyente, donde existen sólo dos o tres empresas monopólicas y dos especies -Pinus radiata y Eucalyptus sp.- dominan la actividad forestal.

\section{BIBLIOGRAFIA}

(1) KIMMINS, J.P. (Hammish). Balancing Act. Environmental issues in forestry. 2nd ed., UBC Press, Vancouver, Canada. 1997. 305 p.

(2) DONOSO, C. Tipos Forestales de los Bosques nativos de Chile. Documento de Trabajo $N^{\circ} 38$ CONAF/FAO/ PNUD FO: DP7CHI/76/003. Santiago, Chile, 1981, $70 \mathrm{p}$.

(3) CONAMA-CONAF. 1999. Catastro y evaluación de recursos vegetacionales nativos de Chile. Informe Nacional con Variables Ambientales. Santiago, Chile, 98 p.

(4) OTERO, L. Aspectos básicos de la certificación forestal. Revista Bosque Nativo (Marzo), 2002, 6 p.

(5) DALY, H. Beyond Growth. Beacon Press, Massachusetts, EE.UU., 1996, 253 p.

(6) INSTITUTO FORESTAL. Estadísticas forestales 1998. Boletín estadístico $\mathrm{N}^{\circ}$ 68. Santiago, Chile, 1999a, 42 p.

(7) INSTITUTO FORESTAL. Exportaciones forestales chilenas. Boletín estadístico N 72. Santiago, Chile, 1999b, $39 \mathrm{p}$.

(8) RIVERA, R., M.E. CRUZ. La realidad forestal chilena. Resultado de Investigaciones $N^{\circ} 15$, GIA, Santiago, Chile. 1983. 45 p.

(9) DESARROLLO HUMANO EN CHILE. Nosotros: Un Desafío Actual. En: Página del PNUD (citado 23 de octubre 2004) Disponible en: http://www.desarrollo humano.cl

(10) Resultados de la VII Encuesta CASEN, 1998. En: Página de MIDEPLAN, División Social, Departamento de Estudios Sociales (citado 25 de octubre 2004). Disponible en: www.mideplan.cl/estudios

(11) El Indice de Desarrollo Humano en la Población Mapuche de la IX Región. 2003. En: Página del PNUD (citado 23 de octubre 2004) Disponible en www.desarrollohumano.cl/otraspub/Pub08/pub08.pdf

(12) MERY, G., S. KENGEN, C. LUJÁN. Forest-based development in Brazil, Chile and Mexico. En: M. Palo, J. Uusivuori y G. Mery (eds.), World Forests, Markets and Policies. Kluwer Academic Publishers, London, UK. 2001. p. 243-252.

(13) Resultados de la VII Encuesta CASEN, 1998. En: Página de MIDEPLAN, División Social, Departamento de Estudios Sociales. Evolución de la pobreza y la indigencia en Chile 1987-1996. Disponible en www.mideplan.cl/ estudios/pobreza01.pdf

(14) Disponible en estadísticas forestales, www.infor.cl (citado 25 de octubre 2003)
(15) SEDJO, R.A., A. GOETZL, S.O. MOFFAT. Sustainability of Temperate Forests. Resources for the Future, Washington DC. 1999. 102 p.

(16) HAMILTON, H. Skogs Gard. Sodra Skogsagarnas. Kristianstad, Suecia. 1990. 245 p.

(17) SKOGSTYRELSEN. Gronare Skog. Ed. Skogstyrelsen Förldag, Jonkoping, Suecia, 1999, 208 p.

(18) O'LAUGHLIN, J., M.G. MESSINA. Swedish forestry and forest policy. Journal of Forestry, 1988, 86 (7): 1720.

(19) GREY, G.W. Finland: Forestry and Technology. Journal of Forestry, 1988, 86(7), 23-26.

(20) BURSCHEL, A. HERNÁNDEZ, M. LOBOS. Leña, una Fuente de Energía Renovable para Chile. Ed. Universitaria, Santiago, Chile, 2003, 171 p.

(21) FERNANDEZ, P. Análisis crítico de la nueva institucionalidad forestal. Revista de Derecho de la Universidad Austral de Chile, Agosto 1998 (Número Especial): 35-41.

(22) EMMANUELI, P. Bosque nativo, antecedentes estadísticos 1985-1994. Corporación Nacional Forestal, Santiago, Chile, 1996. 22 p.

(23) LARA, A., C. DONOSO, J.C. ARAVENA. La conservación del bosque nativo de Chile: problemas y desafios. En: J.J. Armesto, C. Villagrán, and M.K. Arroyo (Eds.): Ecología de los Bosques Nativos de Chile. Editorial Universitaria, Santiago, Chile, 1995, p. 335-362.

(24) OCDE-CEPAL. Evaluaciones de Desempeño Ambiental-Chile. Publicación conjunta de la Organización de Cooperación y Desarrollo Económicos y la Comisión Económica para América Latina y el Caribe. 2005. 246 p.

(25) LARA, A., D. SOTO., J. ARMESTO, P. DONOSO, C. WERNLI, L. NAHUELHUAL, F. SQUEO. Componentes científicos clave para una política nacional sobre usos, servicios y conservación de los bosques nativos chilenos. Libro resultante de la Reunión Científica sobre Bosques Nativos realizada en Valdivia los días 17-18 de julio de 2003. Universidad Austral de Chile. Iniciativa Científica Milenio, 2003, 134 p.

(26) Egresados y Titulados de la Educación Superior. En: Página del Ministerio de Educación de Chile (MINEDUC) (citado el 30 de octubre de 2004). Disponible en: www.mineduc.cl/superior/compendio/egresados.htm

(27) BENEDETTI, S., J. SAAVEDRA. 2002. Estado del arte de la investigación forestal en Chile 1996-2000. En: Página del INFOR (citado 27 de octubre 2004). Disponible en: www.infor.cl

(28) PEREDO, H. El impacto socioambiental en los planes de estudio de ingeniería forestal en las universidades chilenas: diagnóstico y necesidades. Bosque, 1992, 13(2), 9-14.

(29) Estaciones experimentales de las seis distintas macrozonas geográficas del Servicio Forestal de los EE.UU. En: Página del Servicio Forestal (citado 28 de octubre del 2004). Disponible en: www.fs.usda.gov.

(30) TOMBERLIN, D., J. BUONGIORNO. Timber plantations, timber supply, and forest conservation. En: M. Palo, J. Uusivuori y G. Mery (eds.) World Forests, Markets and Policies. Kluwer Academic Publishers, London, UK, 2001, p. 85-94.

(31) SEYMOUR, R., M.L. HUNTER. Principles of ecological forestry. In: M. Hunter (ed.) Maintaining Biodiversity in Forest Ecosystems. Cambridge Univ. Press, Great Britain, 1999 , p. 22-63.

(32) FRANKLIN, J., D.R. BERG, D.A. THORNBURGH, and J. C. TAPPENIER. Alternative silvicultural approaches to timber harvesting: Variable retention harvest systems. En: K. Kohm and J. Franklin (eds.) Creating a Forestry 
for the $21^{\text {st }}$ Century. Island Press, Covelo, California, EE.UU, 1997, p. 111-140.

(33) BOYLE, J.R. Planted forests. Views and viewpoints. New Forests, 1999, 17: 5-9.

(34) SUTTON, W.R.J. The need for planted forests and the example of radiata pine. New Forests, 1995, 10: 95-109.

(35) LARA, A., T.T. VEBLEN. Forest plantations in Chile: a successful model? En:, A. Mather (ed.) Afforestation. Policies, planning, and progress. CRC Press, 1993, p. 118-139.

(36) OTERO, L. Efectos de la sustitución de bosques nativos por plantación de especies exóticas en Chile. En: $L a$ tragedia del bosque chileno. Ed. Ocho Libros, Santiago, 1998, p. 294-298.

(37) MERY, G., M. KANNINEN. Sustainable management of forest plantations and carbon sequestration in Chile. En: M. Palo (ed.), Forest Transitions and Carbon FluxesGlobal Scenarios and Policies. UNU/WIDER, Helsinki, Finland, 1999, p. 74-100.

(38) DRISCOLL, P.V. Comparisons of bird counts from pine forests and indigenous vegetation. Australian Wildlife Research, 1977, 4: 281-288.

(39) COUT, M.N., P.D. GAZE. Effects of plantation forestry on birds in New Zealand. Journal of Applied Ecology, 1984, 21: 795-815.

(40) HUNTER, M.J. Biological diversity. En: M.J. Hunter (ed.), Maintaining biodiversity in forest ecosystems. Cambridge Univ. Press, New York, EE.UU., 1999, p. 3-21.

(41) CANNEL, M.G.R. Environmental impacts of forest monocultures: water use, acidification, wildlife conservation, and carbon storage. New Forests, 1999, 17, 239-262.

(42) MOORE, S.E., H.L. ALLEN. Plantation forestry. En: M.J. Hunter (ed.), Maintaining biodiversity in forest ecosystems. Cambridge Univ. Press, New York, EE.UU., 1999, p. 400-433.

(43) KEENAN, R.J., J.P. (HAMISH) KIMMINS. The ecological effects of clear-cutting. Environmental Review, 1993, 1, 121-144.

(44) BORMANN, D.B., G.E. LIKENS. Pattern and Process in a Forested Ecosystem. New York: Springer-Verlag, 1979, $253 \mathrm{p}$

(45) WALSTAD, J.D., P.J. KUCH. Introduction to forest vegetation management. En: J.D. Walstad and P.J. Kuch (eds.) Forest vegetation management for conifer production. John Wiley and Sons Inc., New York, EE.UU., 1987, p 3-14.

(46) GLADSTONE, W.T., F.T. LEDIG. Reducing pressure on natural forests through high-yield forestry. Forest Ecology and Management, 1990, 35, 69-78.

(47) HUNTER, M.L., A. CALHOUN. A triad approach to land-use allocation. En: R.C. Szaro y D.W. Johnston (eds.), Biodiversity in Managed Landscapes. Oxford University Press, New York, EE.UU., 1996, p. 477-491.

(48) ECHEVERRIA, C., COOMES, D., NEWTON, A., SALAS, J., REY, J. M., A. LARA. 2005 (en Prensa). Rapid fragmentation and deforestation of Chilean Temperate Forests. Biological Conservation.

(49) SCHULZE, E., C. WIRTH, M. HEIMANN. Managing forests after Kyoto. Science. 2002, 289, 2058-2059.

(50) MARLAND, G. 2000. The future role of reforestation in reducing buildup of atmospheric $\mathrm{CO}^{2}$. En T.M.L Wigley and D.S. Schimel (eds.), The Carbon Cycle. Cambridge Univ. Press, N.Y., EE.UU. 2000, p. 190-198.

(51) HOUGHTON, R.A., G.M. WOODWELL. Global climatic change. Scientific American, 1989, 260: 36-47.

(52) WIGLEY, T.M.L., D.S. SCHIMEL. The Carbon Cycle. Cambridge Univ. Press, New York, 2000, 292 p.
(53) HARMON, M.E., W.K. FERREL, J.F. FRANKLIN. Effects of carbon storage of conversion of old-growth forests to young forests. Science, 1990, 247: 699-701.

(54) SKOG, K.E., G.A. NICHOLSON. Carbon cycling through wood products: the role of wood and paper products in carbon sequestration. Forest Products Journal, 1998, 48 (7/8), 75-83.

(55) DE GROOT, R., M. WILSON, R. BOUMANS. A typology for the classification, descriptions and valuation of ecosystem functions, goods and services. Ecological Economics, 2002, 41, 393-408.

(56) DONOSO, C. Bosques templados de Chile y Argentina. Variación, estructura y dinámica. Ed. Universitaria, Santiago, Chile, 1993, 484 p.

(57) ARMESTO, J.J, P. LEÓN LOBOS, M.K. ARROYO. Los bosques templados del sur de Chile y Argentina: una isla biogeográfica. En:, J.J. Armesto, C. Villagrán, and M.K. Arroyo (eds.): Ecología de los Bosques Nativos de Chile. Editorial Universitaria, Santiago, Chile, $1995^{\mathrm{a}}$, p. 23-28.

(58) ARMESTO, J.J., R. ROZZI, C. SMITH-RAMÍREZ, M.T.K. ARROYO. Conservation targets in South American temperate forests. Science, 1998, 282, 12711272.

(59) OLSON, D.M., E. DINERSTEIN. The Global 200: A Representation Approach to Conserving the Earth's Most Biologically Valuable Ecoregions. Conservation Biology, 1998, 12 (3), 502-515.

(60) CINCOTTA, R.P., J. WISNEWSKI, R. ENGELMAN. Human population in the biodiversity hotspots. Nature, 2000, 404, 990-992.

(61) SIMONETTI, J., A. GREZ, R. BUSTAMANTE. El valor de la matriz en la conservación ambiental. Revista Ambiente y Desarrollo, 2002, XVIII, 116-118.

(62) OYARZUN C., L. NAHUELHUAL, D. NUÑEZ. 2005. Los servicios ecosistémicos del bosque templado lluvioso: producción de agua y su valoracion económica. Ambiente y Desarrollo. 20 (3): 88-95.

(63) DONOSO, C., A. LARA. Silvicultura de los bosques nativos de Chile. Ed. Universitaria, Santiago, Chile, 1998, $421 \mathrm{p}$.

(64) SILVA, J., P. DONOSO, A. LARA, C. LITTLE. Estudio de seis años de respuesta al raleo en renovales de roble (Nothofagus obliqua (Mirb.) Oerst.) en el predio Aillapán, comuna de Loncoche, Novena Región. En: Actas Raulí, riqueza de los bosques templados: Silvicultura, Genética e Industria. Simposio Internacional IUFRO, 14-16 de abril, Valdivia, Chile, 2004, 12 p.

(65) MARTINEZ, R., P. DONOSO. Crecimiento de raulí (Nothofagus alpina) en tres diferentes plantaciones en el sector de Riñihue, provincia de Valdivia. En: Actas Raulí, riqueza de los bosques templados: Silvicultura, Genética e Industria. Simposio Internacional IUFRO, 14-16 de abril, Valdivia, Chile, 2004, 9 p.

(66) DONOSO, P., T. MONFIL, L. OTERO, L. BARRALES. Estudio de crecimiento de plantaciones y renovales de especies nativas en el área andina de las provincias de Valdivia y Cautín. Ciencia e Investigación Forestal, 1993, 7(2), 24-42.

(67) DONOSO, P., M.E. GONZALEZ, B. ESCOBAR, I. BASSO, L. OTERO. Viverización y plantación de Raulí, Roble y Coihue en Chile. En: C. Donoso y A. Lara (eds.) Silvicultura de los Bosques Nativos de Chile. Ed. Universitaria, Santiago, Chile, 1999, p. 145-189.

(68) GROSSE, H. Desarrollo de renovales de raulí raleados. Ciencia e Investigación Forestal, 1987, (2), 31-43.

(69) CORTI, D. Caracterización y crecimiento de un renoval de roble (Nothofagus obliqua), lingue (Persea lingue) y 
radal (Lomatia hirsuta) en la comuna de Loncoche, IX Región, 1996, 116 p.

(70) DONOSO, P., C. CABEZAS, A. LAVANDEROS. Estudio comparativo de la estructura y crecimiento de renovales de Coihue (Nothofagus dombeyi) en la precordillera de la Costa y de los Andes de la provincia de Valdivia. Bosque, 2000, 20(2), 9-23.

(71) PETERS, R., M. JOBET, S. AGUIRRE. Compendio de tablas auxiliares para el manejo de plantaciones de pino insigne. Manual $\mathrm{N}^{\circ} 14$, División Silvicultura, Instituto Forestal, Chile, 1985, $140 \mathrm{p}$.

(72) PRADO, J.A. Selección de procedencias de varias especies del género Eucalyptus para la zona centro-sur de Chile. En: Actas manejo Silvícola del género Eucalyptus. Viña del Mar, Chile, 1988, 33 p.
(73) TORO, J. Efecto de la fertilización en el desarrollo inicial de las plantaciones de Eucalyptus en Chile. En: Actas manejo Silvícola del género Eucalyptus. Viña del Mar, Chile, 1988, $13 \mathrm{p}$.

(74) METLA. Finnish Statistical Yearbook of Forestry. Ediciones METLA, Finlandia, 2002, 378 p.

(75) CUBBAGE, F., R. RUBILAR, J. SIRY, P. MAC DONAGH, M. N. BAEZ, J. SAWINSKI JR., A. FERREIRA, V. HOEFLICH, V. MORALES, G. BALMELLI, G. FERREIRA, J. ALVAREZ, P. DONOSO. Comparative Timber Investment Returns for Selected Plantations and Native Forests in South America and the Southern United States. Speech Presented at the Annual Southern Forest Economics Workers (SOFEW) Meeting April 18-20, 2005, Baton Rouge, Louisiana, USA. 30 p. 